\title{
Generalized diffusion equation
}

\author{
Jean Pierre Boon* and James F. Lutskd \\ Center for Nonlinear Phenomena and Complex Systems \\ Université Libre de Bruxelles, 1050 - Bruxelles, Belgium
}

(Dated: April 4, 2018)

\begin{abstract}
Modern analyses of diffusion processes have proposed nonlinear versions of the Fokker-Planck equation to account for non-classical diffusion. These nonlinear equations are usually constructed on a phenomenological basis. Here we introduce a nonlinear transformation by defining the $q$-generating function which, when applied to the intermediate scattering function of classical statistical mechanics, yields, in a mathematically systematic derivation, a generalized form of the advection-diffusion equation in Fourier space. Its solutions are discussed and suggest that the $q$-generating function approach should be a useful tool to generalize classical diffusive transport formulations.
\end{abstract}

PACS numbers: 05.90.+m, 05.60.-k, 05.10.Gg

The development of generalizations of classical statistical mechanical paradigms to describe non-equilibrium systems has become an important area of activity. One of the best known examples of this approach is the socalled "nonextensive" thermodynamics first introduced by Tsallis [1] which is based on a new definition of entropy that as being the $q$-logarithm of the number of states, rather than the natural logarithm in Boltzmann's definition. It has been shown that thermodynamics can be consistantly built using the Tsallis entropy as a starting point [2]. The success of this program has led to the introduction of other "nonextensive" generalizations such as the nonextensive diffusion equation [3]. As in the case of the entropy, these generalizations are usually based on the replacement of a classical, extensive (frequently linear) phenomenological expression by a parameterized, nonextensive (frequently non-linear) expression which reduces to the classical expression for some value of the parameter. In this paper, we show that for the particular case of diffusion it is possible to introduce these generalizations at a more fundamental level. The key point is that statistical mechanical derivations often make use of resummations and ansatzs which can be made "nonextensive " in a natural way since they are intrinsically heuristic.

Processes related to diffusive transport are most commonly measured by scattering methods (photon correlation and thermal neutron spectroscopy) and the spectra are analyzed using the intermediate scattering function [4]

$$
F_{s}(k, t)=\langle\exp [\imath \mathbf{k} \cdot(\mathbf{R}(t)-\mathbf{R}(0))]\rangle
$$

which is the space-Fourier transform of the single particle density correlation function, i.e. the probability that a tagged particle be at position $\mathbf{R}(t)$ at time $t$, given it was at $\mathbf{R}(0)$ at the initial time. In classical statistical mechanics, one shows that this function obeys the usual diffusion - or advection-diffusion - equation in the hydrodynamic limit, i.e. for long wavelengths: $k \ell<<1$, where $\ell$ is the mean free path. This is easily obtained, for in- stance, by the cumulant expansion of $F_{s}(k, t)[4]$. Here we generalize the procedure by defining the $q$-generating function

$$
\mathcal{G}(k, t) \equiv \ln _{q} F_{s}(k, t)=\sum_{j=0}^{\infty} \frac{(\imath \mathbf{k})^{j}}{j !} \cdot \mathrm{K}_{j}(t),
$$

or equivalently the $q$-cumulant expansion

$$
\begin{aligned}
F_{s}(k, t) & =\exp _{q}\left[\sum_{j=0}^{\infty} \frac{(\imath \mathbf{k})^{j}}{j !} \cdot \mathrm{K}_{j}(t)\right] \\
& =\left[1+(1-q) \sum_{j=0}^{\infty} \frac{(\imath \mathbf{k})^{j}}{j !} \cdot \mathrm{K}_{j}(t)\right]^{\frac{1}{1-q}},
\end{aligned}
$$

where $\mathbf{K}_{j}(t)$ are time dependent functions which can be expressed in terms of the spatial moments [4]. Note that in the limit $q \rightarrow 1$, the $q$-log and the $q$-exp reduce to the usual logarithmic and exponential expressions [1]. From (3), we have

$$
\frac{\partial F_{s}(k, t)}{\partial t}=\sum_{j=0}^{\infty} \frac{(\imath \mathbf{k})^{j}}{j !} \cdot\left[\frac{\partial \mathbf{K}_{j}(t)}{\partial t}\right] F_{s}^{q}(k, t),
$$

which is an exact equation that follows from the nonlinear transformation (2). Low $k$ expansion on the r.h.s. of (4) yields

$$
\frac{\partial F_{s}(k, t)}{\partial t}=\left[\imath \mathbf{k} \cdot \frac{\partial \boldsymbol{\kappa}_{1}(t)}{\partial t}-\frac{1}{2} k^{2} \frac{\partial \kappa_{2}(t)}{\partial t}+\ldots\right] F_{s}^{q}(k, t),
$$

where

$$
\frac{\partial \boldsymbol{\kappa}_{1}(t)}{\partial t}=\frac{\partial}{\partial t}\langle[\mathbf{R}(t)-\mathbf{R}(0)]\rangle=\mathbf{u}+\langle\mathbf{v}(t)\rangle=\mathbf{u},
$$

with $\mathbf{u}$ the macroscopic velocity or "drift term ", and

$$
\frac{\partial \kappa_{2}(t)}{\partial t}=\frac{\partial}{\partial t}\left\langle[\mathbf{R}(t)-\mathbf{R}(0)]^{2}\right\rangle=2 D,
$$

where, for simplicity, we have considered isotropic diffusion, with $D$ the self-diffusion coefficient. So to second 
order, as usually considered for the classical hydrodynamic limit, we obtain the nonlinear advection-diffusion equation

$$
\frac{\partial F_{s}(k, t)}{\partial t}=\imath \mathbf{k} \cdot \mathbf{u} F_{s}^{q}(k, t)-D k^{2} F_{s}^{q}(k, t),
$$

or

$$
\frac{1}{1-q} \frac{\partial F_{s}^{1-q}(k, t)}{\partial t}=\imath \mathbf{k} \cdot \mathbf{u}-D k^{2}
$$

The solution for initial condition $F_{s}(k, t=0)=1$ (i.e. a real space delta-function) and for positive times is

$$
\begin{aligned}
F_{s}(k, t) & =\left[1+(1-q)\left(\imath \mathbf{k} \cdot \mathbf{u}-D k^{2}\right) t\right]^{\frac{1}{1-q}} \\
& \equiv \exp _{q}\left[\left(\imath \mathbf{k} \cdot \mathbf{u}-D k^{2}\right) t\right]
\end{aligned}
$$

a form commonly found in nonextensive statistical mechanics [5]. It is clear that, in the limit $q \rightarrow 1$, one retrieves the classical result: $F_{s}(k, t)=e^{\left(\imath \mathbf{k} \cdot \mathbf{u}-D k^{2}\right) t}$.

Equation (8) rewritten as

$$
\frac{\partial F_{s}(k, t)}{\partial t}-\imath \mathbf{k} \cdot \mathbf{u}_{q}(k, t) F_{s}(k, t)=-D k^{2} F_{s}^{q}(k, t),
$$

with $\mathbf{u}_{q}(k, t)=\mathbf{u} F_{s}^{q-1}(k, t)$, can be viewed as the analogue in Fourier space of the porous media equation [6, 7], but in contrast to the latter, the nonlinear advectiondiffusion equation (11) yields generalized, but classical diffusive behavior in real space (i.e. with $r / \sqrt{D t}$ scaling, as we shall see below), and therefore applies to a different class of diffusive transport.

In the absence of flow $(\mathbf{u}=0)$, we call Eq.(8) the $q$ diffusion equation

$$
\frac{\partial F_{s}(k, t)}{\partial t}=-D k^{2} F_{s}^{q}(k, t) .
$$

This generalized equation can be cast into a regular diffusion equation with an effective diffusion coefficient $D_{\text {eff }}$ by using the identification

$$
D_{e f f} \equiv D_{q}=D F_{s}^{q-1}(k, t),
$$

or, substituting the solution given in (10) (with $\mathbf{u}=0$ ) and expanding in $D k^{2} t$ (i.e. for long wavelengths),

$$
D_{q}=D \sum_{j=0}^{\infty}(1-q)^{j}\left(D k^{2} t\right)^{j}
$$

that is $D_{q}=D+$ higher moments $(q, k, t)$, where the higher moments depend on $q$, and also on $k$ and $t$ as for the space- and time-dependent transport coefficients in generalized hydrodynamics [4]. For $q=1$, only the term $j=0$ survives in the sum in (14), and one retrieves the classical diffusion formulation.

We now consider what type of distributions are obtained from the solution to the $q$-diffusion equation (12):

$$
F_{s}(k, t)=\left[1-(1-q) D k^{2} t\right]^{\frac{1}{1-q}} .
$$

As the scattering function must be real and non-negative, the argument in brackets must be positive. This is always realized for $q>1$. If we are to allow $q<1$, then it is necessary to limit the support of the intermediate scattering function by including a step function giving

$$
F_{s}(k, t)=\left[1-(1-q) D k^{2} t\right]^{\frac{1}{1-q}} \Theta\left(1-(1-q) D k^{2} t\right)
$$

where $\Theta(y)=1$ for $y>1$ and $\Theta(y)=0$ otherwise. Substitution of (16) into Eq.(12) shows that (16) satisfies the $q$-diffusion equation since the time-derivative acting on the step function gives a Dirac delta function with a prefactor that goes to zero. Note that in this case, since the intermediate scattering function is a function of $D k^{2} t$, it immediately follows that in real space it is a function of $r^{2} / D t$ so that $\left\langle r^{2}\right\rangle \sim D t$ which corresponds to normal diffusion, albeit with non-classical distribution functions.

In one dimension, inversion of the Fourier transform (15) in the case $q>1$ gives

$$
G_{q>1}(r, t)=\frac{\Gamma^{-1}\left(\frac{1}{q-1}\right)}{\sqrt{\pi} \sqrt{(q-1) D t}}\left(\frac{x}{2}\right)^{\frac{1}{q-1}-\frac{1}{2}} K_{\frac{1}{q-1}-\frac{1}{2}}(x),
$$

where $x=\frac{r}{\sqrt{(q-1) D t}}$, and $K_{\nu}(x)$ is the modified Bessel function of the second kind. The structure of this result should not be too surprising since we know that the general solution to the classical diffusion problem is given in terms of Bessel functions [8]. For some rational values of $q$ it is possible to express the Bessel function in terms of elementary functions in which case the solution assumes the form $p(x) \exp (-x)$ where $p(x)$ is a polynomial in $x$. For instance for $q=3 / 2$, we obtain

$$
G_{3 / 2}(r, t)=\frac{1}{2 \sqrt{2 D t}}(x+1) \exp (-x) .
$$

Figure 1 shows a numerical evaluation of the solutions for various values of $q$, and, as $q$ increases, it does indeed appear to decay as an exponential.

We now show that (17) does give the correct Gaussian limit as $q \rightarrow 1$. We make explicit the dependence on $q$ by writing

$$
K_{\frac{1}{q-1}-\frac{1}{2}}(x)=K_{\frac{1}{q-1}-\frac{1}{2}}\left(\frac{r^{*}}{\sqrt{q-1}}\right),
$$

where $r^{*}=r / \sqrt{D t}$. Introducing $\nu=\frac{1}{q-1}-\frac{1}{2}$ in (17) gives

$$
\begin{aligned}
G_{q>1}(r, t) & =\frac{\Gamma^{-1}\left(\nu+\frac{1}{2}\right)}{\sqrt{\pi} \sqrt{D t}} \sqrt{\nu+\frac{1}{2}}\left(\frac{r^{*}}{2} \sqrt{\nu+\frac{1}{2}}\right)^{\nu} \times \\
& \times K_{\nu}\left(r^{*} \sqrt{\nu+\frac{1}{2}}\right)
\end{aligned}
$$


Using the asymptotic expansion for $\nu \gg 1[9]$

$$
\begin{aligned}
K_{\nu}(\nu z) & \sim \sqrt{\frac{\pi}{2 \nu}}\left(\frac{z}{1+\sqrt{1+z^{2}}}\right)^{-\nu} \frac{1}{\left(1+z^{2}\right)^{1 / 4}} \times \\
& \times \exp \left(-\nu\left(\sqrt{1+z^{2}}\right)\right)
\end{aligned}
$$

with $z=r^{*} \frac{\sqrt{\nu+\frac{1}{2}}}{\nu}$, gives, for fixed $r^{*}$,

$$
\begin{aligned}
& K_{\nu}\left(r^{*} \sqrt{\nu+\frac{1}{2}}\right) \sim \sqrt{\frac{\pi}{2 \nu}}\left(r^{*} \frac{\sqrt{\nu+\frac{1}{2}}}{\nu}\right)^{-\nu} \times \\
& \times \exp \left(\nu \ln 2+\frac{1}{4} r^{* 2}\right) \exp \left(-\nu-\frac{1}{2} r^{* 2}\right) \\
& \sim \sqrt{\frac{\pi}{2 \nu}}\left(r^{*} \frac{\sqrt{\nu+\frac{1}{2}}}{2 \nu}\right)^{-\nu} \exp \left(-\nu-\frac{1}{4} r^{* 2}\right),
\end{aligned}
$$

wherefrom we obtain

$$
G_{q>1}(r, t) \sim \frac{\Gamma^{-1}\left(\nu+\frac{1}{2}\right)}{\sqrt{2 D t}} \nu^{\nu} \exp \left(-\nu-\frac{1}{4} r^{* 2}\right),
$$

and, using Stirling's formula, we find

$$
G_{q>1}(r, t) \sim \frac{1}{2 \sqrt{\pi D t}} \exp \left(-\frac{r^{2}}{4 D t}\right),
$$

as expected. Fig.2 clearly shows that the distribution approaches a Gaussian in the limit $\nu \gg 1$ or $q \rightarrow 1$.

For $q<1$, Fourier inversion of 15 yields

$G_{q<1}(r, t)=\frac{1}{2 \sqrt{\pi}} \frac{\Gamma\left(\frac{1}{1-q}+1\right)}{\sqrt{(1-q) D t}}\left(\frac{x}{2}\right)^{-\frac{1}{1-q}-\frac{1}{2}} J_{\frac{1}{1-q}+\frac{1}{2}}(x)$,

with $x=\frac{r}{\sqrt{(1-q) D t}}$, and where $J_{\nu}$ is the Bessel function of the first kind. Here also, for some values of $q$, this result can be written in terms of elementary functions in the form $l(x) \cos x+m(x) \sin x$ where $l(x)$ and $m(x)$ are polynomials in $x$. As an example, for $q=1 / 2$, we have

$G_{1 / 2}(r, t)=\frac{\pi^{-1}}{\sqrt{2 D t}}\left(\frac{x}{2}\right)^{-5}\left(3 \sin x-3 x \cos x-x^{2} \sin x\right)$.

Figure 2 shows that as $q \rightarrow 1$ the distribution approaches a Gaussian, and Fig. 3 shows $G_{q}(r, t)$ for several values of $q<1$. In this case, we observe that the distribution goes sometimes negative (for small values of $q$ ) and, hence, becomes unphysical. Note that after the distribution first becomes negative, its magnitude is always very small so that in some sense it appears to be approximating a function with finite support. Therefore, we must either (a) restrict the range of allowable values of $q$ to $q \geq 1$, or (b) allow values of $q<1$ with the understanding that the distribution is an approximation to a positive-definite function, perhaps one having finite support. It is worth noting in passing that option (b) is quite commonly used in other areas of statistical mechancs. For example, the Chapman-Enskog solution to the Boltzmann equation results, at all orders, in a distribution which is not positive-definite 10.

It is interesting to ask what conventional diffusion process would yield the above results. Casting the general solutions (17) and (23) into the form of a generalized diffusion equation in real space

$$
\frac{\partial G_{q}(r, t)}{\partial t}=D_{q}(r, t) \frac{\partial^{2} G_{q}(r, t)}{\partial r^{2}} .
$$

gives, for $q>1$,

$$
D_{q>1}(r, t)=D \frac{q-1}{2} \frac{N(r, t)}{M(r, t)},
$$

where, with the notation $\nu=\frac{1}{q-1}-\frac{1}{2}$,

$$
\begin{aligned}
& N(r, t)=\frac{r}{\sqrt{(q-1) D t}} \frac{K_{\nu+1}\left(\frac{r}{\sqrt{(q-1) D t}}\right)}{K_{\nu}\left(\frac{r}{\sqrt{(q-1) D t}}\right)}-1-2 \nu, \\
& M(r, t)=1+\frac{(q-1) D t}{r^{2}}(1-2 \nu)(N(r, t)+1),
\end{aligned}
$$

and, for $q<1$,

$$
D_{q<1}(r, t)=D \frac{1-q}{2} \frac{N^{\prime}(r, t)}{M^{\prime}(r, t)},
$$

where, with the notation $\nu=\frac{1}{1-q}+\frac{1}{2}$,

$$
\begin{aligned}
N^{\prime}(r, t) & =\frac{r}{\sqrt{(1-q) D t}} \frac{J_{\nu+1}\left(\frac{r}{\sqrt{(1-q) D t}}\right)}{J_{\nu}\left(\frac{r}{\sqrt{(1-q) D t}}\right)}-1, \\
M^{\prime}(r, t) & =-1+\frac{(1-q) D t}{r^{2}}(1+2 \nu)\left(N^{\prime}(r, t)+1\right) .
\end{aligned}
$$

Two typical examples (for $q=3 / 2$ and $q=1 / 2$ ) of the generalized diffusion coefficient are 11]

$$
D_{3 / 2}(r, t)=\frac{D^{\prime}}{2}\left(\frac{r}{\sqrt{D^{\prime} t}}+\frac{1}{1-\frac{r}{\sqrt{D^{\prime} t}}}\right),
$$

where $D^{\prime}=\frac{1}{2} D$, and

$$
D_{1 / 2}(r, t)=\frac{D^{\prime}}{2} \frac{x^{2} \sin x+(3-x)(x \cos x-\sin x)}{4 \sin x+\left(\frac{12}{x}-1\right)\left(\cos x-\frac{\sin x}{x}\right)},
$$

with $x=\frac{r}{\sqrt{D^{\prime} t}}$.

Although the diffusion coefficient $D_{q}$ exhibits a generalized form because of its dependence on the distribution function $G_{q}(r, t)$, diffusion remains normal in the sense that $\frac{r}{\sqrt{D t}}$ scaling is preserved as we already observed and 
as can be seen even more explicitly by computing the mean square displacement

$$
\left\langle r^{2}(t)\right\rangle=\int_{0}^{\infty} d r r^{2} G_{q}(r, t)
$$

with $G_{q}(r, t)$ given either by (17) or (23), which, with $r^{*}=r / \sqrt{D t}$, yields

$$
\left\langle r^{2}(t)\right\rangle=D t 2 \int_{0}^{\infty} d r^{*} r^{* 2} G_{q}\left(r^{*}\right)=2 D t
$$

In this paper, we have shown how a standard derivation of the diffusion equation can be generalized into a "nonextensive" form without the introduction of ad hoc modifications. Instead, we simply generalize the ansatz used to resum the expansion of the intermediate scattering function. For an intial condition of a Dirac delta function in real space, the solutions of the resulting diffusion equation in Fourier space take the form of the $q$ exponential which commonly arises in nonextensive statistical mechanics. This solution corresponds to normal diffusion, but with non-classical distributions. Therefore the $q$-generating function approach developed here should be useful to similarly generalize other derivations of diffusive transport.

* Electronic address: jpboon@ulb.ac.be URL: http://poseidon.ulb.ac.be/

† Electronic address: lutsko@ulb.ac.be

[1] C. Tsallis, J. Stat. Phys., 52, 479 (1988).

[2] M. Gell-Mann and C. Tsallis, Nonextensive Entropy (Oxford University Press, New York, 2004).

[3] H.L. Swinney and C. Tsallis, eds. Anomalous Distributions, Nonlinear Dynamics, and Nnextensivity, Physica D, 193 (2004).

[4] See e.g. J.P. Boon and S. Yip, Molecular Hydrodynamics (Dover, New York, 1991); chap. 4.

[5] C. Tsallis, Physica D, 193, 3 (2004), and references therein.

[6] C. Tsallis and D.J. Buckman, Phys. Rev. E, 54, R2197 (1996).

[7] S. Abe and S. Thurner, Anomalous diffusion in view of Einstein's 1905 theory of Brownian motion, arXiv:cond-mat/0411645 to appear in Physica A.

[8] G.B. Arfken and H.J. Weber, Mathematical Methods for Physicists (Academic Press, 5th edition, New York, 2001); chap. 11.

[9] M. Abramowitz and I.A. Stegun, Handbook of Mathematical Functions (NBS Mathematical Series, reprinted by Dover, New York, 1965); chap. 9.

[10] S. Chapman and T.G. Cowling, The mathematical Theory of Non-uniform Gases (Cambridge University Press, London, 1960).

[11] Notice that in both cases the generalized diffusion coefficient exhibits singularities.

\section{FIGURE CAPTIONS}

FIGURE 1 : Distribution $G_{q>1}(r, t)$ as a function of $r^{*}=r / \sqrt{D t}$.

FIGURE 2 : Distribution $G_{q}(r, t)$ as a function of $r^{*}=r / \sqrt{D t}$ for $q=1.05$ and $q=0.95$ compared to the Gaussian solution.

FIGURE 3 : Distribution $G_{q<1}(r, t)$ as a function of $r^{*}=r / \sqrt{D t}$. 

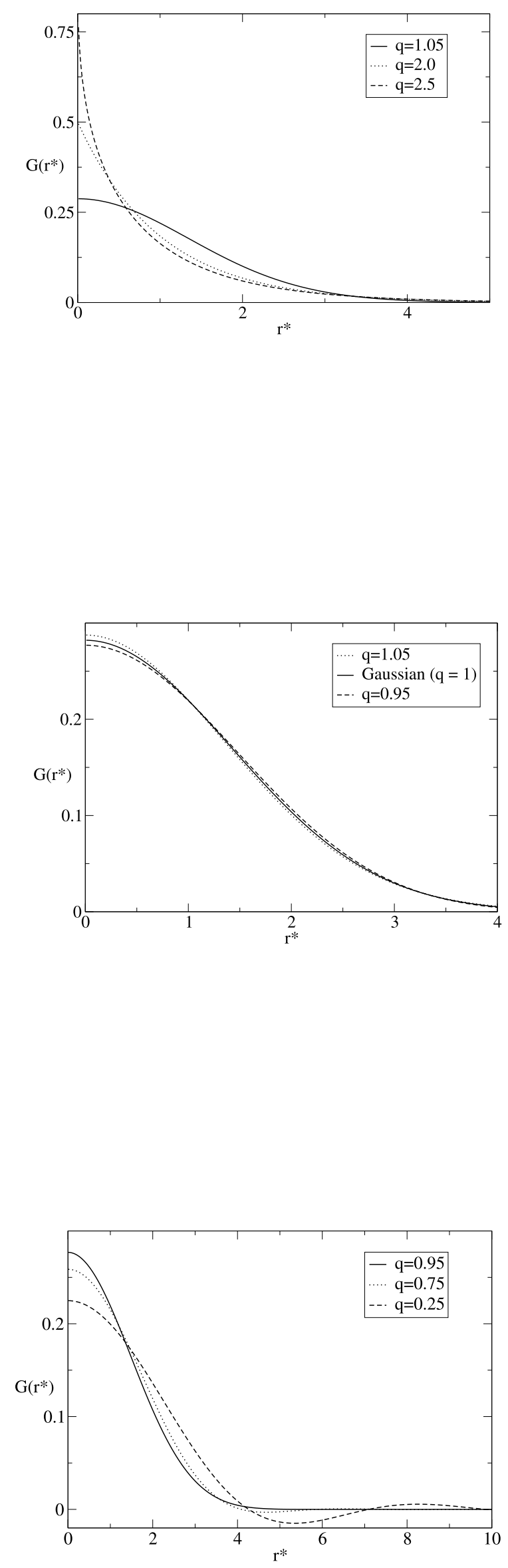